\title{
A MODIFICATION OF GAUSS' METHOD FOR PRELIMINARY DETERMINATION OF A CELESTIAL BODY'S ORBIT
}

\author{
A.A.Bazyey \\ Dpt of Astronomy, Odessa National University \\ Shevchenko Park, Odessa 65014, Ukraine
}

\begin{abstract}
A modification of classical Gauss' method for determination of Keplerian elements from the observed positions is considered in this paper. The modification involves the exhaustive enumeration of all possible orbital plane positions in order to improve the method's reliability. It has been shown that such an approach requires a priori information on the pattern of the celestial body's motion, particularly, whether its motion is direct or retrograde.
\end{abstract}

Key words: orbit determination methods, celestial mechanics

\section{Introduction}

The method for determination of orbital elements of celestial bodies was developed by C.F.Gauss as early as at the beginning of the $19^{\text {th }}$ century when the first asteroids were discovered. Up to the present time, this method has been successfully employed for preliminary orbit determination for both circumsolar and near-Earth orbital motion.

However, the method presents some limitations in its usage (Samotokhin et al., 2014). In particular, the orbital arc whose length is used for calculations should not be too long as in this case difficulties with solution of some equations may emerge. On the other hand, the orbital arc should not be too short as in this case uncertainties associated with observational errors may occur.

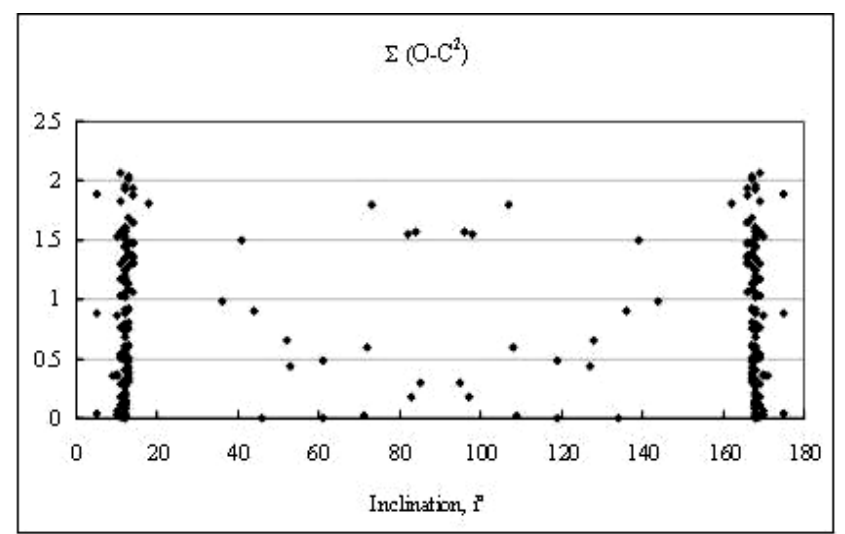

Figure 1. The distribution of the sum of squared differences between the observed and calculated positions of the test celestial body for different orbital inclinations.

\section{Numeral experiment}

These and other limitations of Gauss' method can be overcome by exploiting capabilities of modern computers. Keplerian elements define the size and shape of the conic (the semi-major axis and eccentricity); a celestial body's position at a given instant of time (the time of perigee passage); the conic section orientation within the orbital plane (the argument of perigee); and, finally, the orbital plane position with regard to the reference coordinate system (the inclination and longitude of the ascending node).

At a two-body approximation the orbital plane always intersects the centre of gravity. With this fact the orbital plane position can be independently determined using the method of exhaustive enumeration of all possible values of inclination and longitude of the ascending node (Bondarenko et al., 2014a). All orbital elements can be derived for each pair of elements $i$ and $\Omega$ using Gauss' method for determination of orbital elements from two position vectors and instants of time (Escobal, 1970). Based on these orbital elements, it is possible to define a celestial body's position for the current $i$ and $\Omega$. Using the differences between the observed and calculated in such a manner positions (O-C), it is possible to select the inclination and longitude of the ascending node which define the actual position of the orbital plane. It is evident that it is the minimum difference $(\mathrm{O}-\mathrm{C})$ that corresponds to the actual position of the orbital plane.

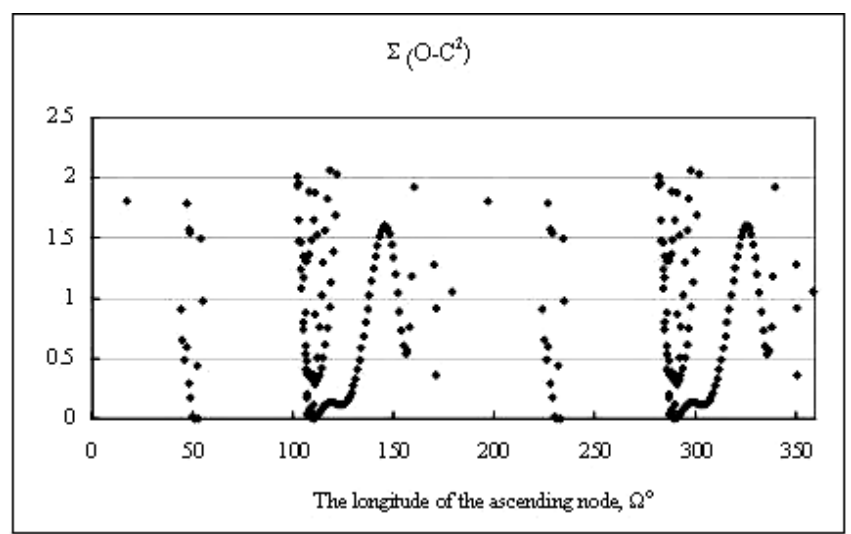

Figure 2. The distribution of the sum of squared differences between the observed and calculated positions of the test celestial body for different longitudes of the ascending node. 
To study the behaviour of the dependence of the sum of squared differences (O-C) on the adopted values of inclination and longitude of the ascending node, we set up a numerical experiment. In this experiment we simulated the search of the position of a test celestial body's orbital plane. Keplerian elements for the heliocentric orbit were selected as follows:

the semi-major axis $a=2.48255 \mathrm{AU}$;

the eccentricity $e=0.15326$;

the time of perigee passage 19.06.2011;

the argument of perigee $\omega=79.325^{\circ}$;

the longitude of the ascending node $\Omega=108.395^{\circ}$; the inclination $i=10.252^{\circ}$.

Using the methods described in (Bondarenko et al., $2014 \mathrm{~b}$ ), we determined the sums of squared differences $(\mathrm{O}-\mathrm{C})$ for all possible pairs of orbital elements, namely the inclination $0 \leq i \leq 180^{\circ}$ and longitude of the ascending node $0 \leq \Omega<360^{\circ}$. From this data set we selected only those values which meet the following requirement:

$$
\Sigma \sqrt{(O-C)^{2}} \leq 1.4^{\prime}
$$

The obtained dependencies are shown in Figs. 1 and 2.

The dependence of $\Sigma(O-C)^{2}$ on the inclination is reflection symmetric with regard to the value $\mathrm{i}=90^{\circ}$, and it exhibits the largest number of the selected values $\Sigma(O-C)^{2}$ near $\mathrm{i}=10.5^{\circ}$ and $\mathrm{i}=169.5^{\circ}$.
The dependence of $\Sigma(O-C)^{2}$ on the longitude of the ascending node is the same for the ranges $0 \leq \Omega<180^{\circ}$ and $180^{\circ} \leq \Omega<360^{\circ}$, and it exhibits the largest number of the selected values $\Sigma(O-C)^{2}$ near $\Omega=108.5^{\circ}$ and $\Omega=288.5^{\circ}$.

\section{Conclusion}

Thus, to ultimately determine the orbital plane using a modified Gauss" method suggested in (Bondarenko et al., 2014a), a priori information on the pattern of the celestial body's motion is required, particularly, whether its motion is direct or retrograde. This requirement is similar to that one for the application of Gauss' method for determination of orbital elements from two position vectors and instants of time (Escobal, 1970).

\section{References}

Bondarenko Yu.S., Vavilov D.E., Medvedev Yu.D.: 2014, Solar System Research, 48, No 3, 212-216.

Bondarenko Yu.S., Vavilov D.E., Medvedev Yu.D.: 2014, Astronomical News Letter, 48, No 3, 1-5.

Escobal P. Methods of Orbit Determination. Moscow: Mir, 1970.

Samotokhin A.S., Khutorovskiy Z.N.: 2014, The Working Papers of M.V.Keldysh Institute of the Applied Mathematics, No 44, 31. 